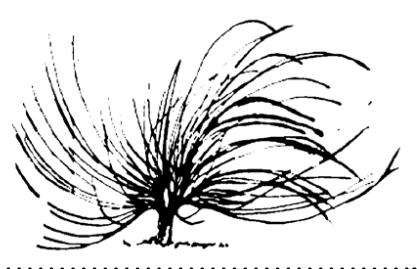

\title{
Políticas educativas que enmarcan la educación dirigida a la población sorda e hipoacúsica en Costa Rica
}

\author{
Almitra Desueza Delgado \\ Ministerio de Educación Pública \\ Costa Rica \\ almitradesueza@gmail.com
}

\begin{abstract}
Resumen
La educación de las personas sordas se encuentra enmarcada y amparada en las leyes que protegen a los sujetos con discapacidad. Este ensayo pretende resaltar las repercusiones de las políticas mundiales, regionales y nacionales que han orientado el desarrollo educativo de la población sorda en Costa Rica, a partir de una revisión bibliográfica en la que se abordarán leyes, decretos internacionales y reglamentos, con los cuales se analizará la situación sociopolítica en la que se inserta la educación de esta población, y se contextualizará el problema en el nivel mundial, latinoamericano y nacional. Las propuestas giran en torno al reconocimiento de la Lengua de Señas Costarricense (LESCO) como lengua materna y las posibilidades, en términos de metodologías educativas, de nuevas prácticas docentes y la integración mayor de la comunidad sorda dentro de su propia educación, para ello es necesario plantearse una sistematización de los avances sociales y culturales involucrados con la comunidad sorda, que permita la reflexión sobre la educación de las personas sordas.
\end{abstract}

\section{(1) 9}

Recibido: 21 de febrero de 2020. Aprobado: 1 de junio de 2021

http://dx.doi.org/10.15359/rep.16-2.1

1 Estudiante del Doctorado en Educación de la Universidad de Costa Rica. M. Sc. en Comunicación. Licenciada en Docencia. Bachiller en Filología Clásica. Docente de Español, Ministerio de Educación Pública, Costa Rica. 
Palabras clave: Adecuaciones curriculares, Costa Rica, desarrollo educativo, discapacidad, educación, políticas educativas, sordos e hipoacúsicos

\begin{abstract}
The education of deaf people is framed and supported by the laws that protect subjects with disabilities. This essay aims to highlight the repercussions of global, regional, and national policies that have guided the educational development of the deaf population in Costa Rica based on a bibliographic review, in which laws, international decrees, and regulations will be addressed. With them, the socio-political situation in which the education of this population is inserted will be analyzed, and the problem will be contextualized on global, Latin American, and national levels. The proposals revolve around the recognition of Costa Rican Sign Language (LESCO, for its acronym in Spanish) as a mother tongue and the possibilities in terms of educational methodologies, new teaching practices and the greater integration of the deaf community within their own education. For this, it is necessary to consider a systematization of the social and cultural advances involved with the deaf community, which allows a reflection on the education of deaf people.
\end{abstract}

Keywords: Costa Rica, curricular accommodations, deaf, education, educational development, educational policies, and hard of hearing

\title{
Introducción
}

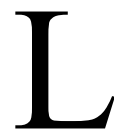

a educación dirigida a las personas sordas e hipoacúsicas fue desarrollada, inicialmente, dentro del paradigma de la discapacidad. En la última década esa visión ha cambiado al incorporarse el paradigma de la integración, con un enfoque desde la inclusión, en donde se da un énfasis más comunicativo, centrado en el lenguaje de señas y el español como segunda lengua; no obstante, esto parece evidenciar poca cohesión y coherencia entre los planes de estudios y las políticas nacionales e internacionales, lo cual ha incidido directamente en la formación de estos individuos. El objetivo del presente ensayo es resaltar las repercusiones de las políticas mundiales, regionales 
y nacionales que han orientado el desarrollo educativo de la población sorda en Costa Rica, a partir de una revisión bibliográfica en la que se abordarán leyes, decretos internacionales y reglamentos, con los cuales se analizará la situación sociopolítica en la que se inserta la educación de esta población, lo que permitirá hacer una contextualización mundial, latinoamericana y nacional, para analizar, desde el marco legislativo, el estado actual de la educación de las personas sordas e hipoacúsicas, con miras a proponer un enfoque paradigmático alternativo, consecuente con los convenios internacionales, las demandas mundiales tanto en el nivel laboral como educativo y las necesidades particulares de los sujetos.

Se utiliza el término de persona sorda en lugar de persona no oyente, porque la sordera es mucho más que una condición física, es una condición cultural. La comunidad sorda se identifica a sí misma con esa terminología, con una serie de parámetros sociales, con costumbres específicas que trascienden la ausencia o presencia de la audición. La comunidad sorda también está compuesta de personas oyentes, las cuales se identifican con los valores y las costumbres de las personas sordas y se consideran parte de esta comunidad.

\section{Las políticas internacionales con respecto a la educación de las per- sonas sordas}

La visibilización de las personas con algún tipo de discapacidad comienza a gestarse en el nivel internacional en 1990 con la Declaración Mundial Sobre Educación Para Todos, con la cual se propone la incorporación de todos los sujetos dentro del ámbito educativo, de tal manera que el artículo primero expone que "cada persona -niño, joven o adulto- deberá estar en condiciones de aprovechar las oportunidades educativas ofrecidas para satisfacer sus necesidades básicas de aprendizaje" (UNESCO, 1990, p. 3). Para ello, se debe contemplar las herramientas básicas de aprendizaje, las necesidades especiales de los individuos y los contextos en los que se desarrollan, por eso, se propone mejorar los entornos donde ocurre el aprendizaje, así como extender las coberturas de la educación especialmente al "empeñarse activamente en modificar las desigualdades en materia de educación y suprimir las discriminaciones en las posibilidades de aprendizaje de los grupos desasistidos" (UNESCO, 1990, p. 5). En general, los 10 artículos de la declaración, mencionada anteriormente, proponen reformas 
a los sistemas educativos e invitan a los gobiernos a comprometerse a hacer de la educación un proceso inclusivo y universal.

Posteriormente, en 1994, se establecen las Normas Uniformes sobre la Igualdad de Oportunidades para Personas con Discapacidad, por parte de la Organización de Naciones Unidas (ONU), en la cual se define que una persona con discapacidad o minusvalía (este término ya no está en uso desde la década de los 90, pero expresaba la connotación semántica hacia las poblaciones diversas), es aquella que tiene algún tipo de limitaciones funcionales o deficiencia física, mental o sensorial (ONU, 1994). Con el fin de salvaguardar la integridad de esta población, se proponen una serie de medidas de rehabilitación, seguridad médica, seguridad alimentaria, seguridad familiar, acceso a la educación, entre otras. Con respecto a la educación;

Los Estados deben reconocer el principio de la igualdad de oportunidades de educación en los niveles primario, secundario y superior para los niños, los jóvenes y los adultos con discapacidad en entornos integrados, y deben velar porque la educación de las personas con discapacidad constituya una parte integrante del sistema de enseñanza. (ONU, 1994, p. 16)

Para ello, se ejecutaron propuestas y planes de acción, por ejemplo, en Costa Rica se fortalecieron las aulas integradas; sin embargo, la atención a esta población siguió llevándose a cabo desde la ausencia, o sea, desde el compromiso cognitivo o físico que presentara el sujeto.

No obstante, en el mismo año, se da la Declaración de Salamanca, UNESCO, 1994, con la cual se cambia el concepto de discapacidad y minusvalía por Necesidades Educativas Especiales (NEE), se reconoce la capacidad del individuo de aprender sin importar el compromiso físico o cognitivo que presente, también la necesidad de promover centros educativos que respeten la diversidad y la diferencia, especialmente de las personas que tienen necesidades educativas especiales, con el fin de combatir todas las actitudes discriminatorias hacia las poblaciones más vulnerabilizadas.

Con esta declaración, se da una transformación importante en la conceptualización y el abordaje de las personas con NEE, primero porque se apuesta a la educación como medio de independencia e integración social, de tal manera que el individuo deja de ser un estorbo 
familiar o una carga para el Estado, y se convierte en un sujeto productivo de la sociedad; en segundo lugar, porque se le devuelven los derechos inherentes a todo ser humano, según la Carta de Derechos de los Seres Humanos promulgada por la ONU en 1945, y en tercer lugar, porque ya no se busca la homogenización de los sujetos, sino que se persigue la optimización del individuo, a partir de la particularidad.

En 1999, la Organización de Estados Americanos (OEA) realiza la Convención Interamericana para la Eliminación de Todas las Formas de Discriminación Contra las Personas con Discapacidad, su objetivo primordial era ir disminuyendo, poco a poco, los diferentes tipos de discriminación que sufrían las personas con algún tipo de compromiso cognitivo o físico, tanto en el nivel de salud, educación, empleo, derechos sexuales y reproductivos, familia, patrimonio cultural, patrimonio económico, entre otros.

La lucha por el cambio semiótico o interpretativo, sobre las personas con alguna situación o compromiso físico o mental ha sido constante; sin embargo, mi crítica ronda en que se vuelve al concepto de discapacidad como deficiencia o ausencia de algo, lo que supone la incompletud del sujeto y, por lo tanto, la incapacidad del mismo para desarrollarse de manera autónoma e integral dentro de la sociedad.

En el 2009, la Agencia Europea para el Desarrollo de la Educación del Alumnado con Necesidades Educativas Especiales divulga los Principios Fundamentales para la Promoción de la Calidad de la Educación Inclusiva. Con este, se articula el concepto de inclusión en lugar de integración. Para ello, la multiculturalidad y el reconocimiento de los grupos de sujetos con algún tipo de compromiso físico o cognitivo, como sujetos pertenecientes a un grupo cultural específico con características socioculturales particulares, los cuales participan activamente de los hechos educativos, a través de distintos mecanismos de acceso, que permiten mejorar la calidad de la educación, de esta forma se encuentran estrechamente vinculadas la calidad, el acceso y la inclusión en el ámbito educativo.

En octubre del 2014, se presenta la Declaración de Lima: Educación para Todos en América Latina y el Caribe, en la cual se destaca como prioridad el desarrollo de "estrategias comprehensivas de educación para fortalecer su participación en la educación y la culminación de ciclos educativos, a través de programas de educación inter, multiculturales y multilingües, y programas que respondan a las 
diversas necesidades educativas" (UNESCO, 2015, p. 2), pues se busca incentivar la adquisición de habilidades para la vida en todos los estudiantes, independientemente de sus particularidades, con el fin de que puedan tener una vida digna. Esta declaración exigía un compromiso por parte de los Estados miembros, con la finalidad de alcanzar excelencia en la calidad brindada, en especial la pública, para con ello fortalecer la democracia.

La Organización de las Naciones Unidas para la Educación, la Ciencia y la Cultura (UNESCO), junto con otros entes internacionales, realiza en el 2015, en Incheon, Corea, un Foro mundial sobre la Educación, con el cual se proyectan hasta el 2030. En este, se plantea la necesidad de ofrecer educación de excelencia a todas las personas, para incrementar la calidad y esperanza de vida en todos los seres humanos, al reconocer la pluralidad cultural, la diversidad lingüística y de entornos sociales, así como las diferentes formas de acceder al conocimiento y aprender. Para ello, se establece una agenda de trabajo común en la cual los Estados se comprometen a trabajar colaborativamente para "garantizar una educación inclusiva, equitativa y de calidad y promover oportunidades de aprendizaje durante toda la vida para todos" (UNESCO, 2015, p. 15).

En enero del 2015, se llevó a cabo la reunión regional de Ministros de Educación de América Latina y el Caribe, como parte de las actividades de monitoreo propuestas en Incheon y se dio la Declaración de Buenos Aires, con la cual los países miembros se comprometieron a "desarrollar políticas inclusivas con miras a la mejora de la calidad y la pertinencia de la educación que incidan en todos los actores del sistema educativo (...) asegurando el éxito escolar en los ciclos sucesivos" (UNESCO, 2017, p. 10), con ello ya no solo se prevé la inclusión de todos los sujetos dentro de los sistemas educativos, sino que además se integra a la comunidad educativa, al cuerpo docente y a todos los actores que intervienen en el proceso educativo como parte fundamental del éxito del mismo.

Por otra parte, en el caso de la educación de las personas sordas e hipoacúsicas, tiene un rol importante la lengua de señas, pues ella es la que convierte a este grupo humano, en particular, en una comunidad. En el ámbito internacional, es importante rescatar la Declaración Universal de la UNESCO sobre la diversidad cultural, en la cual se expone, en los objetivos 4 y 5 del anexo II que, como parte de la diversidad cultural 
es función de la UNESCO; "salvaguardar el patrimonio lingüístico de la humanidad y apoya la expresión, la creación y la difusión en el mayor número de lenguas", así como "fomentar la diversidad lingüística - respetando la lengua materna - en todos los niveles de enseñanza, dondequiera que sea posible, y estimular el aprendizaje de varios idiomas desde la edad más temprana (UNESCO, 1995, p. 3). Con lo cual, se pretende conservar los legados que las diversas comunidades lingüísticas producen.

Asimismo, con la Declaración Universal de Derechos Lingüisticos, en 1996, se reconoce el derecho de las distintas comunidades lingüísticas para acceder al aprendizaje y a los modelos de enseñanza, en su lengua materna, así como de poseer en esta, los diferentes recursos que requieren para desarrollarse en sociedad (Comité de seguimiento de la Declaración Universal de Derechos Lingüísticos, 1998). En el caso de la comunidad sorda en Costa Rica, en el 2012, la Asamblea Legislativa aprobó la Ley 9049, Ley de Reconocimiento de Lenguaje de Señas Costarricense (LESCO) como lengua materna. Con ello, el Estado se compromete a impartir las clases en LESCO, así como todos los servicios de educación (planes de estudios, elementos evaluativos, investigación, difusión, entre otros) a través del Ministerio de Educación Pública (MEP).

Además, la Asamblea Legislativa de Costa Rica en 1996 divulga la Ley 7600, Ley de Igualdad de Oportunidades para las Personas con Discapacidad, en concordancia con los acuerdos internacionales y como parte del avance en derechos humanos. Bajo esta ley, se amparan, en principio los sujetos sordos, pues la misma les asegura la formulación de programas que se adapten a sus necesidades, así como la adaptación de servicios de apoyo, materiales didácticos y su inclusión en el aula regular, ya que en el artículo 18 dice que "las personas con necesidades educativas especiales podrán recibir su educación en el Sistema Educativo Regular" (Asamblea Legislativa de Costa Rica, 1996, p. 14). En 1999, se crea la Ley 7948, Convención Interamericana para la Eliminación de Todas las Formas de Discriminación contra las Personas con Discapacidad, en correspondencia con la convención de la OEA; asimismo en el 2008 se crea la Ley 8661, Convención sobre los Derechos de las Personas con Discapacidad y su Protocolo Facultativo, en concordancia con la convención de la ONU. Finalmente, en 
el 2016 se crea la Ley 9379, Ley para la Promoción de la Autonomía Personal de las Personas con Discapacidad.

Bajo ese marco jurídico, se cobijan todas las personas sordas e hipoacúsicas del país, lo que se supone les asegura el acceso a una educación de calidad, en la cual se utilice el LESCO como medio principal de comunicación y se desarrollen los hábitos culturales en los que se desenvuelve esta población. El problema está en algunas discordancias que se encuentran dentro del sistema educativo, por ejemplo, el acceso al LESCO desde temprana edad, una evaluación educativa acorde con los métodos de enseñanza, entre otros.

La mayor cantidad de niños sordos y niñas sordas pertenecen a familias oyentes, eso significa que el proceso temprano de adquisición de la lengua no se da de la misma manera que con un niño oyente con padres oyentes, sino que el niño o la niña desarrolla un sistema de comunicación particular con su cuidador o cuidadora, no replicado por otros, ya que todavía no pertenece a una comunidad lingüística. En segundo lugar, cuando el individuo ingresa al sistema de escolarización adquiere la LESCO de manera "natural", sin instrucción previa, sino a partir de la interacción con sus docentes, sus compañeros y compañeras. En tercer lugar, no hay dentro del currículo de las personas sordas ningún curso de gramática de LESCO, el énfasis se pone en la enseñanza del español como segunda lengua.

Debido a que el nacimiento de una persona sorda no está delimitado a una zona geográfica específica, sino que puede ubicarse en cualquier parte del país, eso complica la designación de la comunidad sorda a partir de un espacio, lo cual significa que la cantidad de personas sordas varía de un lugar a otro. De esta manera, la cercanía entre los individuos facilitará o no, la concreción de centros de enseñanza de la LESCO, ya que son los adultos los que transmiten el conocimiento a los niños y las niñas, eso pone en desventaja a los sujetos que se encuentran más aislados.

Unido a esto, se encuentra la escolarización. Es muy común que los individuos que se encuentren aislados de otros sujetos sordos se ubiquen en zonas rurales, con poco acceso a la tecnología y a las facilidades urbanas. También, es común que los centros educativos no tengan un docente de educación especial con énfasis en audición y lenguaje, por lo que usualmente lo que tienen es un docente itinerante, que llega unas horas por semana, en las que debe aclarar todas las dudas de los estudiantes. 
Esta problemática se une al hecho de que los niños y las niñas aprenden LESCO con los docentes y el equipo que trabaja con ellos, así como con sus pares. Entre más aislado se encuentre el individuo, menos posibilidades tendrá de desarrollar, de forma adecuada, la LESCO, lo que supone un proceso de aislamiento mayor y menores posibilidades de comunicación. No obstante, también existen centros educativos especializados en la enseñanza de niños sordos y niñas sordas, como por ejemplo la Escuela Fernando Centeno Güell ubicada en Goicochea y el centro Educativo Dr. Carlos Sáenz Herrera en Cartago. Y, en secundaria, están las aulas de sordos ubicadas en algunos centros educativos, los programas de educación técnica, así como la inclusión en el aula regular.

No existe un programa de enseñanza sobre la gramática y la estructura del LESCO, pues estos se centran en la enseñanza del español como segunda lengua. El problema es que, si no existe una adecuada recepción de la información en donde el código sea común y utilizado por todos los involucrados, lo que se genera es mucha distorsión en el mensaje, dando cabida a la interpretación equivocada del mensaje. No se puede esperar que un estudiante entienda una segunda lengua si no tiene el dominio de su lengua materna, por lo menos el dominio hablante. Cuando los programas para la enseñanza de estudiantes sordos se centran en la enseñanza de la segunda lengua, significa que se desecha la posibilidad de que alguno de ellos no domine su lengua materna, o en otras palabras, se asume que todos los estudiantes sordos tienen como lengua materna el LESCO y obvian el hecho de que más que una lengua materna, es una lengua de crianza.

La lengua materna es aquella que se habla en el seno familiar, es el centro de comunicación de un grupo específico, los adultos se la transmiten a los niños con todos los modismos, expresiones lingüísticas, ademanes, gesticulación, entre otros. Por otra parte, la lengua de crianza es una lengua transmitida por un grupo externo a la familia, en tiempos distintos a los de socialización familiar, y que, normalmente, depende de reglas exógenas. Cuando hago referencia a que la mayoría de los niños sordos y las niñas sordas tienen la LESCO como lengua de crianza es porque estos nacen en hogares oyentes, que, por lo general, no conocen la LESCO y la aprenden a través de personas exógenas, quienes son las que también interactúan con los niños y con las niñas, pero en protocolos sociales distintos a los familiares y casi siempre en edades preescolares o escolares. 
Es por ello que es necesario fortalecer la lengua de crianza con actividades sociales más próximas a los niños y a las niñas, con una estructuración mayor de la enseñanza de la LESCO, que permita el incremento del vocabulario, facilite el uso de la gramática y genere dominio de esta lengua, para poder utilizarla, posteriormente, como base para el aprendizaje de la segunda lengua. Eso también implica que la aplicación de instrumentos de evaluación debería hacerse primero en LESCO, por lo menos en primer ciclo; en segundo ciclo, se puede hacer un híbrido evaluativo, así como en tercer ciclo y, en Educación Diversificada, podría evaluarse en español escrito. El problema está en que los instrumentos evaluativos están centrados en la producción escrita, lo que resulta ser la segunda lengua de los sujetos sordos.

La evaluación no corresponde a la metodología empleada comúnmente en las clases. Las clases son más visoespaciales, están centradas en la lengua de señas, en la gesticulación y en la articulación del sujeto dentro del espacio, con un apoyo importante de imágenes; pero las evaluaciones cuantitativas están centradas en las formas escritas, lo que pone en desventaja a los estudiantes, especialmente en las pruebas estandarizadas como las de bachillerato o las de alto impacto, como exámenes de admisión a centros de educación superior, pues se les evalúa a los estudiantes en la segunda lengua no en la lengua de crianza o en la lengua materna y ello podría incidir en el error de comprensión. El fracaso continuo de los sujetos en las pruebas de bachillerato puede disminuir las expectativas a futuro que estos tenga de sí mismos.

Dentro de las repercusiones que tienen las actuales medidas de aprendizaje para la población sorda e hipoacúsica en Costa Rica, está un acceso real a la educación superior mucho menor, primero por la dificultad que tienen para aprobar los exámenes de admisión a los centros educativos públicos y, en segundo lugar, por la falta de recursos económicos para acceder a centros de educación superior privados. Esto influye directamente en el acceso al trabajo, pues entre menores sean sus habilidades y su capacidad de producción, menor será la posibilidad que tengan para insertarse en los mercados laborales.

Las políticas nacionales e internacionales existen. La legislación costarricense asegura que el MEP es el ente garante de proteger la calidad de educación de las personas con discapacidad (en donde entra la población sorda), así como los encargados de velar por que la LESCO sea la lengua con la que se enseñe a los sujetos sordos; el problema 
radica en la aplicación de estas políticas. En primer lugar, porque no existen estudios longitudinales ni comparativos, que de forma empírica, sustenten los planes de estudios utilizados con la población sorda. Tampoco existen políticas sociales que busquen la cohesión de esta comunidad en el nivel nacional o que fomenten el intercambio de conocimientos entre los docentes sordos y los docentes oyentes que trabajan con esta población.

\section{Conclusiones}

En síntesis, el mundo entero lleva aproximadamente 30 años haciendo esfuerzos importantes por visibilizar las poblaciones con necesidades educativas especiales, buscando garantizarles un acceso adecuado a una educación con calidad que les permita ser autónomos y productivos. Para ello, se ha tenido que pasar por varias etapas de desmitificación conceptual y se han abierto los debates nacionales e internacionales, lo que permitió pasar del concepto de minusvalía, al de discapacitado y al concepto de compromiso cognitivo o fisico, el cual se maneja en la actualidad. En el campo de la educación, se han logrado avances importantes, se ha pasado del aula integrada a la inclusión de los individuos en el aula regular; falta mucho camino por recorrer, especialmente en relación con la disminución del tamaño de los grupos y la preparación docente.

Con respecto a la población sorda, el reconocimiento de la LESCO como lengua materna abrió las puertas a otras metodologías educativas, a nuevas prácticas docentes y a una integración mayor de la comunidad sorda dentro de su propia educación. Sin embargo, no existe un plan claro sobre cómo debería ser la educación para esta población y la LESCO se encuentra invisibilizada en las prácticas evaluativas, así como materia de estudio dentro de la malla curricular. Falta evidencia empírica que permita responder a la necesidad de fortalecer la enseñanza del español como segunda lengua o la enseñanza de la LESCO como parte de las estrategias metacognitivas que permitan la comprensión del español escrito.

Particularmente, considero importante plantearse la creación de un bloque de aprendizaje de LESCO para estudiantes sordos, su gramática y vocabulario, de tal manera que los niños desde el preescolar hasta noveno año vayan ampliando su comprensión de esta lengua de crianza hasta transformarla en una lengua materna, lo cual favorecería los 
procesos de adquisición de otras lenguas. También, me parece importante analizar la necesidad de evaluar en LESCO, inclusive las pruebas de bachillerato; en el caso de Español, habría que preguntarse cuál es el nivel de comprensión lectora y de producción textual que se busca en una persona sorda y, a partir de allí, tomar las medidas necesarias.

\section{Referencias}

Agencia Europea para el Desarrollo de la Educación del Alumnado con Necesidades Educativas Especiales. (2009). Principios fundamentales para la promoción de la calidad de la educación inclusiva. Lifelong learning programme.

Asamblea Legislativa de Costa Rica. (1996). Ley 7600 Igualdad de oportunidades para las personas con discapacidad y su reglamento. La Gaceta.

Asamblea Legislativa de Costa Rica. (1999). Ley 7948 Convención Interamericana para la eliminación de todas las formas de discriminación contra las personas con discapacidad. La Gaceta.

Asamblea Legislativa de Costa Rica. (2008). Ley 8661 Convención sobre los derechos de las personas con discapacidad y su protocolo facultativo. La Gaceta.

Asamblea Legislativa de Costa Rica. (2012). Ley de reconocimiento del Lenguaje de señas costarricense (LESCO) como lengua materna, Ley 9049. La Gaceta.

Comité de seguimiento de la Declaración Universal de Derechos Lingüísticos. (1998). Declaración Universal de Derechos Lingüisticos. Comité de seguimiento de la Declaración Universal de Derechos Lingüísticos.

OEA. (1999). Convención Interamericana para la eliminación de todas las formas de discriminación contra las personas con discapacidad. OEA.

ONU. (1994). Normas uniformes sobre la igualdad de oportunidades para las personas con discapacidad. ONU.

UNESCO. (1990). Declaración mundial sobre educación para todos y marco de acción para satisfacer las necesidades básicas de aprendizaje. UNESCO.

UNESCO. (1994). Declaración de Salamanca y marco de acción para las necesidades educativas especiales. UNESCO. 
UNESCO. (1995). Declaración universal de la UNESCO sobre la diversidad cultural. UNESCO.

UNESCO. (2014). Educación para todos en América Latina y el Caribe: balance y desafios post-2015. UNESCO.

UNESCO. (2015). Educación 2030: Declaración de Incheon y Marco de Acción. UNESCO.

UNESCO. (2017). Educación 2030: Declaración de Buenos Aires. UNESCO. 
